\title{
ON THE VIBRATION OF ELASTIC BODIES HAVING TIME-DEPENDENT BOUNDARY CONDITIONS*
}

\author{
BY \\ J. G. BERRY AND P. M. NAGHDI \\ University of Michigan
}

1. Introduction. In the classical treatment of the small vibrations of elastic bodies, the displacements are assumed to have the form ${ }^{1}$

$$
U_{i}(x, t)=u_{i}^{n}(x) q_{n}(t), \quad\left(x \equiv x_{1}, x_{2}, x_{3}\right),
$$

where the $u_{i}^{n}(x)$ are the so-called normal modes and the $q_{n}(t)$ are the generalized coordinates. The boundary conditions, which are homogeneous, serve to determine the mode shapes and the secular equation governing the natural frequencies. If, however, the boundary conditions are non-homogeneous, the classical method usually fails since, in general, the boundary conditions and the displacements involve different functions of time. ${ }^{2}$

Among the various methods which may be employed in the study of the motion of a vibrating elastic body having time dependent boundary conditions, the integral transform techniques and the use of Lagrange's equations, ${ }^{3}$ for certain problems, are perhaps most familiar. An alternative approach is to transform the original problem into an equivalent forced vibration problem with homogeneous boundary conditions; this approach is adopted here. The basic idea involving the transformation of variables so as to remove the non-homogeneous boundary conditions, is well known [1, p. 277]. However, this procedure, in connection with vibration of elastic bodies having time dependent boundary conditions, was first used by Mindlin and Goodman [2] in discussing the motion of beams and later by Herrmann [3] for problems of rods.

The present paper contains a method of solution for the motion of any finite, vibrating, elastic body having arbitrary time-dependent boundary conditions and initial conditions. The method used involves a transformation of the type employed by Mindlin and Goodman [2]. However, the resulting forced vibration problem is discussed without reference to stress strain relations and therefore the procedure is quite different from that contained in [2] or [3]. Since the stress strain relations are not explicitly specified the results are readily applicable to all linear theories of elasticity including the special theories of rods, beams, plates and shells; in each case, it is only necessary to introduce

\footnotetext{
*Received March 15, 1955; revised manuscript received June 7, 1955. The results presented in this paper were obtained in the course of research sponsored by the Office of Naval Research under Contract Nonr-1224 (01) with the University of Michigan.

${ }^{1}$ Throughout this paper, the indices (subscripts or superscripts) $i$ and $j$ take the values 1,2 , and 3 only. The indices $m$ and $n$ take all positive integer values, and all other indices take values as specified upon introduction. The indices $i$ and $j$ are reserved for quantities which have tensor character while the indices $m$ and $n$ are used exclusively to denote normal modes. Repeated indices imply summation over all the values the indices may take. Whenever one of the repeated indices is placed in parenthesis, the convention is suspended for that index.

'An important exception occurs when the boundary conditions are simple harmonic functions of time and only the steady state solution is sought. In this case, no difficulties are encountered.

'Some remarks concerning the use of Lagrange's equations are given in Sec. 5.
} 
the appropriate stress strain relations. This, together with some of the features of the procedure, is illustrated by means of an example in Sec. 4.

2. Statement of the problem. Let $\mathrm{V}$ and $\mathrm{S}$ be the volume and the boundary surface of a region $R$ (not necessarily simply connected) occupied by an isotropic, elastic medium." Further, let $U_{i}, \sigma_{i j}, X_{i}$ and $\rho$ denote, respectively, the displacement vector, the stress tensor, the body forces, and the mass density all referred to a set of rectangular cartesian coordinates $x_{i}$. Then, the equations of motion for the medium are

$$
\sigma_{i, j}+X_{i}=\rho \partial^{2} U_{i} / \partial t^{2}
$$

where comma denotes partial differentiation with respect to the space variables.

Let $B_{k}$ denote a system of spatial differential operators such that when they are applied to appropriate components of the displacement vector (written as $B_{k}\left[U_{1}, U_{2}\right.$, $\left.U_{3}\right]$ ), these quantities take on prescribed values on the boundary $S$ and will be assumed to have the product form $g(x) f(t)$. Thus, if there are $p$ boundary conditions, $r(r \leq p)$ of which are non-zero, the boundary conditions may be written as

$$
B_{k}\left[U_{1}, U_{2}, U_{3}\right]=g_{k}(x) f^{l}(t) \delta_{(k)}^{l} \quad \text { on } S,
$$

where $\delta_{k}^{l}$ is the Kronecker delta, and the indices $k$ and $l$ have the range $k=1,2,3 \cdots p$, $l=1,2,3 \cdots r$. It is important to note that the notation $B_{k}\left[U_{1}, U_{2}, U_{3}\right]$ does not imply that the operators $B_{k}$ are applied to the displacement vector $U_{i}$; the $B_{k}$ are applied only to appropriate components of $U_{i}$ [see for example Eqs. (23)].

We now wish to determine a set of displacement functions $U_{i}$ which satisfy the equations of motion throughout $R$, the boundary conditions on $S$, and the initial conditions in $R$ for all $t \leq 0$.

3. Development of the method. We introduce the transformation

$$
U_{i}(x, t)=v_{i}^{l}(x) f^{l}(t)+\xi_{i}(x, t),
$$

where $v_{i}^{l}$ denotes the $l$ th displacement vector which will be defined presently. If we now require that the functions $v_{i}^{l}$ take the values $B_{k}\left[v_{1}^{l}, v_{2}^{l}, v_{3}^{l}\right]=g_{k} \delta_{(k)}^{l}$ on $S$, it then follows from (3) and (4) that the displacements $\xi_{i}$ must satisfy the homogeneous boundary conditions $B_{k}\left[\xi_{1}, \xi_{2}, \xi_{3}\right]=0$ on $S$.

A suitable choice of $v_{i}^{l}$ may be made in various ways. Mindlin and Goodman [2] represented $v_{i}^{l}$ as polynomials with arbitrary constants which were then adjusted to give the desired results. A more general method, which reduces to that of [2] for certain classes of problems, is to choose $v_{i}^{l}$ to be the displacements which satisfy the following system of statical problems:

$$
\begin{aligned}
\tau_{i j, j}^{l} & =0 \quad \text { in } R, \\
B_{k}\left[v_{1}^{l}, v_{2}^{l}, v_{3}^{l}\right] & =g_{k}(x) \delta_{(k)}^{l} \quad \text { on } S,
\end{aligned}
$$

where $\tau_{i j}^{l}$ is that portion of $\sigma_{i j}$ which is obtained from the displacements $v_{i}^{l}$. Equations (5) represent a system of $(l)$ separate problems, each of which has been obtained from the original problem [Eqs. (2) and (3)] by setting $X_{i}=0, \partial^{2} U_{i} / \partial t^{2}=0$, replacing $\sigma_{i j}$ by $\tau_{i j}^{l}$, and setting all $f(t)=0$ except that $f^{l}(t)=1$ for each value of $l$ in turn. Thus, each

"Actually, the medium need not be assumed to be isotropic. In fact, the subsequent analysis is valid whenever the medium possesses a strain energy function which is positive definite. 
of the $r$ sets of $v_{i}^{l}$ satisfy the same equilibrium equations but different boundary conditions. In the special case where the boundary conditions are all displacement conditions (no surface tractions), this procedure coincides with that used by Herrmann [3].

Due to the manner in which the boundary conditions appear in (5), it is possible that the region $R$ is not in statical equilibrium. For example, consider $R$ to be a beam pinned at one end only and acted upon by an oscillating couple such that the beam rotates about the pin. The corresponding statical problem characterized by (5) is a beam pinned at one end only and acted upon by a constant couple; it is evident that the beam is not in statical equilibrium. This difficulty is easily resolved if we interpret statical equilibrium according to D'Alembert's principle. Thus, if the region $R$ is undergoing rigid body motions due to the tractions on $S$, it is sufficient to add to the first of (5) those body forces necessary to insure equilibrium. We shall denote these body forces by $Q_{i}^{l}(x)$. In general, there will be one set for each of the $(l)$ separate problems. The necessity for introducing the forces $Q_{i}^{l}$ is really a consequence of the manner in which the $v_{i}^{l}$ were chosen.

In what follows, it will be assumed that the displacements $v_{i}^{l}$ are known. In view of the character of the transformation (4), the displacements $\xi_{i}$ are the solutions of a forced vibration problem with homogeneous boundary conditions. In keeping with the classical approach to such problems, we assume $\xi_{i}$ to have the form

$$
\xi_{i}(x, t)=u_{i}^{n}(x) q_{n}(t),
$$

where $u_{i}^{n}$ are the normal modes of the associated free vibration problem and the functions $q_{n}(t)$ are the generalized coordinates, as yet undetermined.

Before proceeding further, it is advantageous to deduce certain relations associated with the normal modes. To this end, let $\eta_{i}(x, t)$ in the form

$$
\eta_{i}=\sum_{n} u_{i}^{n}(x) \exp \left[(-1)^{1 / 2} \omega_{(n)} t\right]
$$

be the displacements which satisfy

$$
\begin{array}{cc}
S_{i j, j}=\rho \partial^{2} \eta_{i} / \partial t^{2} & \text { in } R, \\
B_{k}\left[\eta_{1}, \eta_{2}, \eta_{3}\right]=0 & \text { on } S .
\end{array}
$$

Equations (8), which serve to determine the normal modes and the natural frequencies $\omega_{n}$, are obtained from (2) and (3) by putting $X_{i}=0$, replacing $U_{i}$ by $\eta_{i}, \sigma_{i j}$ by $S_{i j}$, and setting all $f^{l}(t)=0$.

The kinetic energy associated with $\eta_{i}$ is given by

$$
T=\frac{1}{2} \int_{V} \rho\left(\partial \eta_{i} / \partial t\right)\left(\partial \eta_{i} / \partial t\right) d V
$$

and in view of (7), the maximum kinetic energy corresponding to the $n$th normal mode is

$$
T_{n}^{\prime}=\frac{1}{2} \omega_{n}^{2} \int_{V} \rho u_{i}^{(n)} u_{i}^{(n)} d V
$$

The normal modes, in addition to (9b), also satisfy Clebsch's [4] orthogonality conditions

$$
\int_{V} \rho u_{i}^{n} u_{i}^{m} d V=0, \quad(n \neq m) .
$$


With the aid of (7), the first of (8) can be written as

$$
\sum_{n} S_{i, i}^{n}=-\rho \omega_{n}^{2} u_{i}^{n}
$$

where $S_{i j}$ is that portion of the stress tensor $\sigma_{i j}$ which is determined from the $n$th normal mode. If Eqs. (11a) are now multiplied by $u_{i}^{m}$ and integrated throughout $R$, there results for each $n$

$$
\int_{V} u_{i}^{m} S_{i, i}^{m} d V=-\omega_{(n)}^{2} \int_{V} \rho u_{i}^{n} u_{i}^{m} d V
$$

which, by (9b) and (10), becomes

$$
\begin{array}{rlrl}
\int_{\nabla} u_{i}^{m} S_{i i, i}^{n} d V & =0 & \text { for } n \neq m \\
& =-2 T_{n}^{\prime} \text { for } n=m .
\end{array}
$$

As will be seen shortly, Eqs. (12) play an important role in the determination of the generalized coordinates.

Substituting (4) into (2) and with the aid of (5), (6), and (8), Eq. (2) will appear as

$$
\tau_{i, i, i}^{l} f^{l}(t)+S_{i j, i}^{n} q_{n}(t)+X_{i}=\rho v_{i}^{l} \partial^{2} f^{l} / \partial t^{2}+\rho u_{i}^{n} \partial^{2} q_{n} / \partial t^{2},
$$

where, according to (5), the first term on the left hand side of (13) is either zero or - $Q_{i}^{l} f^{l}(t)$, depending on whether the region $R$ is in statical equilibrium or not. The differential equation governing $q_{n}(t)$ is now obtained by multiplying (13) by $u_{i}^{m}$, integrating over the volume $V$ of $R$, and using (12) with the result

$$
\frac{\partial^{2} q_{n}}{\partial t^{2}}+\omega_{(n)}^{2} q_{n}=\int_{V} u_{i}^{n}\left[X_{i}-\rho v_{i}^{l} \frac{\partial^{2} f^{l}}{\partial t^{2}}-Q_{i}^{l} f^{l}\right] d V / \int_{V} \rho u_{i}^{(n)} u_{i}^{(n)} d V
$$

the general solution of which is

$$
q_{n}(t)=A_{n} \sin \omega_{(n)} t+B_{n} \cos \omega_{(n)} t+\frac{1}{\omega_{(n)}} \int_{0}^{t} P_{n}(\tau) \sin \omega_{(n)}(t-\tau) d \tau .
$$

In (15) $A_{n}$ and $B_{n}$ are constants and $P_{n}(t)$ represents the right hand side of (14). If the initial conditions are

$$
U_{i}(x, 0)=U_{i 0}(x), \quad \partial U_{i}(x, 0) / \partial t=U_{i 0}^{\prime}(x),
$$

then, with the aid of (10), the following expressions for $A_{n}$ and $B_{n}$ are readily deduced:

$$
\begin{gathered}
A_{n}=\int_{V} \rho u_{i}^{n}\left[U_{i 0}^{\prime}-v_{i}^{l}\left(\frac{\partial f^{l}(0)}{\partial t}\right)\right] d V / \omega_{(n)} \int_{V} \rho u_{i}^{(n)} u_{i}^{(n)} d V, \\
B_{n}=\int_{V} \rho u_{i}^{n}\left[U_{i 0}-v_{i}^{l} f^{l}(0)\right] d V / \int_{V} \rho u_{i}^{(n)} u_{i}^{(n)} d V .
\end{gathered}
$$

This completes the formal solution of the problem. By way of recapitulation, the steps leading to the solution of the problem under consideration are: (a) assume that the 
displacements are given by (4) and (6); (b) determine the displacements $v_{i}^{2}$ by solving the system of statical problems defined by (5); (c) solve the free vibration problem obtained by setting $X_{i}=0$ and $f^{l}(t)=0$ in (2) and (3); and then (d) evaluate the integrals appearing in (15) and (17).

It may be noted that whenever the boundary conditions involve simple harmonic functions of time, the above procedure can be modified slightly. In such cases it is convenient to choose $v_{i}^{l}$ to be a set of steady state solutions corresponding to each time dependent boundary condition. This modification simplifies the results somewhat.

4. An example. Consider a rectangular beam of length $L$, thickness $h$, and width $b$. Let the beam be referred to a set of cartesian axes $x_{i}$ with the length measured along $x_{1}$, while $x_{2}$ is positive downward; the origin of $x_{i}$ is placed at the centroid of the left end section. The beam is assumed to be simply supported and is driven by a stepmoment at the left end; the step-moment is the product of a constant moment $M_{0}$ and a unit step function in time.

The equations which govern the motion of such a beam are obtained by assuming that the non-vanishing stresses are $\sigma_{11}$ and $\sigma_{12}$ and that these are functions of $x_{1}, x_{2}$ and $t$ only. We also assume that the bending stress $\sigma_{11}$ varies linearly across the thickness. A set of displacements which are consistent with the last assumption, are

$$
U_{1}=x_{2} \psi\left(x_{1}, t\right), \quad U_{2}=w\left(x_{1}, t\right) .
$$

The equilibrium equations and the stress displacement relations which may be readily obtained from the general equations of elasticity (see, for example, Ref. [5]) are

$$
\begin{aligned}
\frac{\partial M}{\partial x_{1}}-Q & =\rho I \frac{\partial^{2} \psi}{\partial t^{2}}, \\
\frac{\partial Q}{\partial x_{1}} & =\rho A \frac{\partial^{2} w}{\partial t^{2}},
\end{aligned}
$$

and

$$
M=E I \frac{\partial \psi}{\partial x_{1}}, \quad Q=\mu k A\left(\psi+\frac{\partial w}{\partial x_{1}}\right),
$$

where the bending moment $M$ and the shear force $Q$ are defined by

$$
M=b \int_{-\Lambda / 2}^{+\Lambda / 2} \sigma_{11} x_{2} d x_{2}, \quad Q=b \int_{-\Lambda / 2}^{+\Lambda / 2} \sigma_{12} d x_{2} .
$$

In (19) and (20), $E$ is Young's modulus, $\mu$ is the shear modulus, $I=b h^{3} / 12, A$ is the cross-sectional area, and $k$ is a constant whose physical significance has recently been discussed by Mindlin and Deresiewicz [6], but need not concern us here. The substitution of (20) into (19) yields the following two differential equations for $\psi$ and $w$.

$$
\begin{aligned}
E I \frac{\partial^{2} \psi}{\partial x_{1}^{2}}-\mu k A\left(\psi+\frac{\partial w}{\partial x_{1}}\right) & =\rho I \frac{\partial^{2} \psi}{\partial t^{2}}, \\
\mu k A\left(\frac{\partial \psi}{\partial x_{1}}+\frac{\partial^{2} w}{\partial x_{1}^{2}}\right) & =\rho A \frac{\partial^{2} w}{\partial t^{2}},
\end{aligned}
$$


which are generally attributed to Timoshenko [7]. Also for the example stated, the boundary conditions are

$$
\begin{gathered}
B_{1}\left[x_{2} \psi, w\right] \equiv w(0, t)=0, \quad B_{2}\left[x_{2} \psi, w\right] \equiv E I \frac{\partial \psi(0, t)}{\partial x_{1}}=M_{0} f(t), \\
B_{3}\left[x_{2} \psi, w\right] \equiv w(L, t)=0, \quad B_{4}\left[x_{2} \psi, w\right] \equiv E I \frac{\partial \psi(L, t)}{\partial x_{1}}=0 ; \\
f(t)= \begin{cases}0 & \text { for } t<0 \\
1 & \text { for } t \geq 0 .\end{cases}
\end{gathered}
$$

According to Eqs. (4) and (6) we take $\psi$ and $w$ as

$$
\begin{aligned}
x_{2} \psi & =v_{1} f(t)+u_{1}^{n} q_{n}(t), \\
w & =v_{2} f(t)+u_{2}^{n} q_{n}(t),
\end{aligned}
$$

where $v_{1}$ and $v_{2}$ satisfy the equilibrium equations

$$
\begin{gathered}
E I \frac{d^{2}\left(x_{2}^{-1} v_{1}\right)}{d x_{1}^{2}}-\mu k A\left\{\left(x_{2}^{-1} v_{1}\right)+\frac{d v_{2}}{d x_{1}}\right\}=0, \\
\frac{d\left(x_{2}^{-1} v_{1}\right)}{d x_{1}}+\frac{d^{2} v_{2}}{d x_{1}^{2}}=0,
\end{gathered}
$$

and the boundary conditions

$$
\begin{array}{ll}
B_{1}\left[v_{1}, v_{2}\right] \equiv v_{2}(0)=0, & B_{2}\left[v_{1}, v_{2}\right] \equiv E I \frac{d\left(x_{2}^{-1} v_{1}(0)\right)}{d x_{1}}=M_{0}, \\
B_{3}\left[v_{1}, v_{2}\right] \equiv v_{3}(L)=0, & B_{4}\left[v_{1}, v_{2}\right] \equiv \frac{d\left(x_{2}^{-1} v_{1}(L)\right)}{d x_{1}}=0 .
\end{array}
$$

It can be readily verified that the following expressions for $v_{1}$ and $v_{2}$ satisfy (25) and (26):

where

$$
\begin{aligned}
\frac{E I}{M_{0} L} x_{2}^{-1} v_{1} & =-\frac{x_{1}^{2}}{2 L^{2}}+\frac{x_{1}}{L}-\frac{I}{L^{2} A}\left(\frac{c_{1}}{c_{2}}\right)^{2}-\frac{1}{3}, \\
\frac{E I}{M_{0} L} \cdot v_{2} & =\frac{x_{1}^{3}}{6 L^{2}}-\frac{x_{1}^{2}}{2 L}+\frac{x_{1}}{3},
\end{aligned}
$$

$$
c_{1}^{2}=\frac{E}{\rho} \quad \text { and } \quad c_{2}^{2}=k \frac{\mu}{\rho} .
$$

The normal modes $u_{1}^{n}$ and $u_{2}^{n}$ which satisfy the equations ${ }^{5}$

$$
\begin{aligned}
E I \frac{d^{2}\left(x_{2}^{-1} u_{1}^{n}\right)}{d x_{1}^{2}}-\mu k A\left\{\left(x_{2}^{-1} u_{1}^{n}\right)+\frac{d u_{2}^{n}}{d x_{1}}\right\} & =-\rho I \omega_{n}^{2}\left(x_{2}^{-1} u_{1}^{n}\right), \\
\mu k A\left\{\frac{d\left(x_{2}^{-1} u_{1}^{n}\right)}{d x_{1}}+\frac{d^{2} u_{2}^{n}}{d x_{1}^{2}}\right\} & =-\rho A \omega_{n}^{2} u_{2}^{n},
\end{aligned}
$$

'In the remainder of this section the summation convention is suspended for the index $n$ only. 
and the boundary conditions

$$
\begin{gathered}
u_{2}^{n}(0)=u_{2}^{n}(L)=0 \\
\frac{d\left(x_{2}^{-1} u_{1}^{n}(0)\right)}{d x_{1}}=\frac{d\left(x_{2}^{-1} u_{1}^{n}(L)\right)}{d x_{1}}=0
\end{gathered}
$$

are

$$
x_{2}^{-1} u_{1}^{n}=C_{n} \cos \frac{n \pi x_{1}}{L}, \quad u_{2}^{n}=D_{n} \sin \frac{n \pi x_{1}}{L} .
$$

Substitution of (30) into (28) yields

from which

$$
\begin{gathered}
C_{n}\left[E I\left(\frac{n \pi}{L}\right)^{2}+\mu k A-\rho I \omega_{n}^{2}\right]+D_{n}\left[\mu k A \frac{n \pi}{L}\right]=0, \\
C_{n}\left[\frac{n \pi}{L}\right]+D_{n}\left[\left(\frac{n \pi}{L}\right)^{2}-\left(\frac{\omega_{n}}{C_{2}}\right)^{2}\right]=0,
\end{gathered}
$$

or

$$
\omega_{n}^{2}=\frac{1}{2}\left[\left(\frac{n \pi}{L}\right)^{2}\left(c_{1}^{2}+c_{2}^{2}\right)+\frac{A}{I} c_{2}^{2}\right] \pm\left\{\left[\left(\frac{n \pi}{L}\right)^{2}\left(c_{1}^{2}+c_{2}^{2}\right)+\frac{A}{I} c_{2}^{2}\right]^{2}-4\left(\frac{n \pi}{L}\right)^{4} c_{1}^{2} c_{2}^{2}\right\}^{1 / 2} .
$$

There are two frequencies for each mode shape which correspond to the presence of two non-zero components of the displacement; they will be denoted by $\omega_{n 1}$ and $\omega_{n 2}$.

If we return to (28) and (29), we may observe that these equations are also satisfied by

$$
x_{2}^{-1} u_{1}^{0}=C_{0}, \quad u_{2}^{0}=0
$$

provided $\omega_{0}^{2}=A / I c_{2}^{2} ; u_{1}^{0}$ is the so-called "thickness shear mode". The notation is not meant to imply that the lowest frequency is $\omega_{0}$; in fact, this frequency is quite large.

When (31) are solved for the ratio of the constants, the normal modes take their final form as

$$
x_{2}^{-1} u_{1}^{n}=\cos \frac{n \pi x_{1}}{L}, \quad u_{2}^{n}=\frac{(n \pi L) c_{2}^{2}}{\omega_{n}^{2} L^{2}-\left(n \pi c_{2}\right)^{2}} \sin \frac{n \pi x_{1}}{L}
$$

and the shear mode becomes $x_{2}^{-1} u_{1}^{0}=\cos \omega_{0} t$. The solution will be complete when the integrals appearing in (14) and (17) are evaluated. Since $f(t)$ is the unit step function $\partial^{2} f(t) / \partial t^{2}=0, \partial f(0) / \partial t=0$, and $f(0)=1$. Hence, $P_{n}(t)=0$, and if we choose the initial conditions to be zero, the only non-vanishing constants are $B_{n}$ given by

$$
B_{n}=-\int_{0}^{L}\left[\frac{h^{2}}{12} u_{1}^{n} v_{1}+u_{2}^{n} v_{2}\right] d x_{1} / \int_{0}^{L}\left[\frac{h^{2}}{12}\left(u_{1}^{n}\right)^{2}+\left(u_{2}^{n}\right)^{2}\right] d x_{1} .
$$

Upon evaluating the above, we find after some manipulation

$$
\begin{aligned}
& B_{n 1}=-2\left(\omega_{n 1}^{2}-\omega_{n 2}^{2}\right)^{-1}\left[\left(\frac{\omega_{n 2}}{n \pi}\right)^{2}-\left(\frac{c_{1}}{L}\right)^{2}\right], \\
& B_{n 2}=2\left(\omega_{n 1}^{2}-\omega_{n 2}^{2}\right)^{-1}\left[\left(\frac{\omega_{n 1}}{n \pi}\right)^{2}-\left(\frac{c_{1}}{L}\right)^{2}\right] .
\end{aligned}
$$


Finally, combining all of the above results, we have

$$
\begin{aligned}
\frac{E I}{M_{0} L} \psi=\frac{x_{1}}{L}-\frac{x_{1}^{2}}{2 L^{2}} & -\frac{I}{L^{2} A}\left(\frac{c_{1}}{c_{2}}\right)^{2}-\frac{1}{3}+\frac{I c_{1}}{L^{2} A c_{2}} \cos \omega_{0} t \\
& -2 \sum_{n=1}^{\infty}\left(\omega_{n 1}^{2}-\omega_{n 2}^{2}\right)^{-1} \cos \frac{n \pi x_{1}}{L}\left\{\left[\left(\frac{\omega_{n 2}}{n \pi}\right)^{2}-\left(\frac{c_{1}}{L}\right)^{2}\right] \cos \omega_{n 1} t\right. \\
& \left.-\left[\left(\frac{\omega_{n 1}}{n \pi}\right)^{2}-\left(\frac{c_{1}}{L}\right)^{2}\right] \cos \omega_{n 2} t\right\}, \\
\frac{E I}{M_{0} L} w=\frac{x_{1}}{3}-\frac{x_{1}^{2}}{2 L}+ & \frac{x_{1}^{3}}{6 L^{3}}+2 \frac{c_{1}^{2} c_{2}^{2}}{L^{2}} \sum_{n=1}^{\infty}\left(\omega_{n 1}^{2}-\omega_{n 2}^{2}\right)^{-1} n \pi \sin \frac{n \pi x_{1}}{L}\left[\frac{\cos \omega_{n 1} t}{\omega_{n 1}^{2}}-\frac{\cos \omega_{n 2} t}{\omega_{n 2}^{2}}\right] .
\end{aligned}
$$

This example has been solved previously by Leonard and Budiansky [8], using the method of Laplace transform. Equations (37) may be regarded as the "indicial admittance" for this boundary driven problem. We could combine these results with Duhamel's integral to obtain the solution for a much more general forcing function.

5. Concluding remark. In the case where the non-homogeneous boundary conditions involve surface tractions only, it is possible to obtain a formal solution by using Lagrange's equations of motion, such a solution is assumed to have the form given by (1) while the surface tractions are incorporated into $q_{n}(t)$ through the generalized work. Thus the solution obtained in this manner will never satisfy the required boundary conditions of the problem, as may be seen from the fact that $u_{i}^{n}$ satisfy equations of the type specified by (8).

Since the normal modes satisfy the homogeneous boundary conditions, it becomes necessary to establish the validity of the solution by showing, through a limiting process, that the solution satisfies the non-homogeneous boundary conditions as the boundary is approached from the interior of $R$. This process, quite often, requires that the solution also be obtained by another method, such as the one given in this paper.

It may be mentioned that a similar situation may also arise in connection with the use of the integral transform methods when the solution is expressed in terms of a convolution integral.

\section{REFERENCES}

1. R. Courant and D. Hilbert, Methods of mathematical physics, Vol. I, Interscience, 1953

2. R. D. Mindlin and L. E. Goodman, Beam vibrations with time-dependent boundary conditions, J. Appl. Mech. 17, 377-380 (1950)

3. G. Herrmann, Forced motion of elastic rods, J. Appl. Mech. 21, 221-224, (1954)

4. A. Clebsch, Theorie de l'elasticité des corps solides, translated from the German by B. de Saint-Venant and Flamant, Paris 1883, p. 129. Also see A. E. H. Love, Mathematical theory of elasticity, Dover, 1944, p. 180

5. R. D. Mindlin, Influence of rotary inertia and shear on flexural motions of isotropic elastic plates, J. Appl. Mech. 18, 31-38 (1951)

6. R. D. Mindlin and H. Deresiewicz, Timoshenko's shear coefficient for flexural vibrations of beams, Proc. Second U.S. Natl. Congr. Appl. Mech. 175-178 (1955)

7. S. P. Timoshenko, On the correction for shear in the differential equations for transverse vibrations of prismatic bars, Phil. Mag. ser. 6, 41, 744-746 (1921)

8. R. W. Leonard and B. Budianski, On traveling waves in beams, NACA TN 2874 (1953) 